\title{
Disrupted physical pain sensation by social exclusion in women with dysmenorrhea
}

This article was published in the following Dove Press journal: Journal of Pain Research

\section{WenJun $\mathrm{Yu}^{1,2}$ \\ Fang Guan' \\ Li Fu' \\ Cheng Long ${ }^{3}$ \\ Li Yang'}

'School of Psychology and Center for Studies of Psychological Application, South China Normal University, Guangzhou, People's Republic of China; ${ }^{2}$ College of Education, JingGangShan University, Ji'an, People's Republic of China; ${ }^{3}$ School of Life Sciences, South China Normal University, Guangzhou, People's Republic of China
Correspondence: Li Yang School of Psychology and Center for Studies of Psychological Application, South China Normal University, 55 W Zhongshan Blvd, Tianhe District, Guangzhou, 51063I, People's Republic of China

Tel +86208521 0369

Email yang_li@m.scnu.edu.cn
Purpose: Dysmenorrhea (DM), which involves both acute and chronic pain, is associated with abnormalities in pain modulation. Previous studies have shown that social pain relies on some of the same neural regions that process physical pain, highlighting a possible physical-social pain overlap. However, evidence suggesting that social pain modulates the sensitivity to physical pain remains controversial. The present study aimed to assess the effects of social pain manipulation on sensitivity to physical pain in DM sufferers and healthy female controls (HC).

Materials and methods: Fifty-nine otherwise healthy patients with DM and $55 \mathrm{HC}$ matched for age, education, calendar age, and gynecological age were randomly assigned to inclusion or exclusion conditions of Cyberball, a virtual ball tossing game used to induce social pain (social exclusion). Pain threshold and pain tolerance in response to nociceptive pressure and cold stimuli were assessed before and after the study condition.

Results: In response to cold stimulation, pain threshold and tolerance significantly reduced in DM compared to HC participants in the inclusion condition, indicating increased pain sensitivity in DM group. However, exclusion increased the pain threshold and tolerance compared to inclusion in DM but decreased pain threshold and tolerance in HC. Neither inclusion nor exclusion altered pressure pain sensitivity in DM, but, after social exclusion, DM participants were more fearful of being evaluated unfavorably. No significant differences were observed in self-esteem scores between DM and HC after both inclusion and exclusion.

Conclusion: We observed altered pain sensation in DM participants in response to social pain manipulation, suggesting that DM not only impacts menstruation but also modulates the perception of pain more generally, especially its affective processes. The present study suggests that the effect of social pain on pain sensation and certain psychometric properties depends on previous pain experience, implicating a reciprocal influence of social and physical pain processes. Keywords: dysmenorrhea, Cyberball, pain modulation, pain sensitivity, physical pain, social pain, social exclusion

\section{Introduction}

Dysmenorrhea (DM), defined as painful menstrual cramps of uterine origin, is a prevailing gynecological problem in women of reproductive age. ${ }^{1}$ Despite its high prevalence, DM is usually poorly treated and even neglected by health professionals, such that women who suffer from DM often accept it as a normal part of the menstrual cycle. ${ }^{2}$ Notably, long-term DM is considered to be a unique pain condition involving both acute and chronic pain, ${ }^{3}$ and later in life, DM often co-occurs with chronic pain conditions, including painful bladder syndrome, fibromyalgia, chronic headache, low back pain, and many others. ${ }^{2,3}$ Susceptibility to the subsequent development of various 
functional disorders has been associated with maladaptive descending pain modulatory systems. ${ }^{3-5}$ Correspondingly, DM sufferers exhibit widespread reductions in both somatic ${ }^{6}$ and visceral ${ }^{7}$ hyperalgesia, ${ }^{4}$ suggesting enhanced pain perception in women with DM compared to healthy female controls (HC). ${ }^{8}$ DM negatively affects women on social relationships, such as family relationships and friendships. ${ }^{9}$ At the clinical level, DM showed increased loneliness compared with $\mathrm{HC}$, which in turn is associated with increased menstrual pain. ${ }^{10}$ Exclusion feeling, one type social pain, ${ }^{11}$ when induced by informing participants that they will end up lonely later in life, was associated with a change in pain sensitivity. ${ }^{12}$

Social pain is the unpleasant experience associated with actual or potential damage to one's sense of social connection or social value, due to social rejection, exclusion, negative social evaluation or loss, etc. ${ }^{13}$ Social pain has been mostly studied using Cyberball, a paradigm based on a virtual balltossing game, where participants believe that they are playing with other real participants. ${ }^{14}$ In fact, they are playing with a preprogrammed computer that enrolls the participant into one of two conditions: a condition in which the participant is socially included (inclusion condition) and one in which he or she is excluded from the game (exclusion condition). ${ }^{15,16}$ There is growing recognition that social and physical pain share many of the same neural and psychological mechanisms. ${ }^{15}$ Emerging evidence shows that social pain relies on some of the same neural regions that process physical pain, highlighting a possible network overlap of physical and social pain, ${ }^{11}$ resulting in heightened perception of social exclusion due to physical injury; conversely, perception of social exclusion can trigger feelings of physical pain. ${ }^{17}$ Furthermore, baseline sensitivity to physical pain can be used to predict sensitivity to social exclusion, and, in turn, heightened social distress increases sensitivity to physical pain. ${ }^{18}$ It is worth noting, however, that previous studies of the impact of social exclusion on pain sensitivity showed inconsistent results. Some showed that social exclusion led to increased emotional- and physical-pain sensitivity, whereas others indicated that exclusion caused numbing of emotional and physical pain. ${ }^{12,18,19}$ Hypersensitivity or numbness have been explained by the "severity hypothesis", which asserts that "minor" social injuries should trigger painful (hypersensitive) reactions, whereas "major" social injuries should result in numbing (insensitive) responses. ${ }^{19}$ Negative social encounters are the "minor" social injuries. Based on the severity hypothesis, mildly negative social encounters would trigger painful (hypersensitive) reactions. However, existing research has demonstrated that mildly negative social encoun- ters can reduce physical pain sensitivity as well. ${ }^{20}$ Thus, the abovementioned severity hypothesis does not fully explain the discrepancy among previous studies. We speculate that there may be other factors affecting the influence of social pain on the physical pain sensitivity.

Given that the neuronal networks underlying social pain and physical pain overlap, we hypothesized that individual experience, especially individual pain experience, would differentially modulate the effects of social pain on the sensitivity of physical pain. Modulation of physical pain following social manipulation during a Cyberball game has not yet been studied for a condition, such as DM, which exhibits a combination of acute and chronic pain. To this end, the current study investigated the modulatory role of social pain on the sensation of acute physical pain in DM and HC individuals.

\section{Materials and methods \\ Participants}

Participants in this study were recruited from the local college or university by advertisement and word of mouth. We first recruited women through platforms online, and by posting posters around the campus. Then, the enrolled women were asked to go to the research center to fill out the DM self-rating scale. The researcher then selects suspected DM and healthy women based on the scores of the scale. All suspected DM women were requested to go to gynecological clinic and finally diagnosed by gynecologist. The diagnostic criteria for DM have been defined by the American College of Obstetricians. The following inclusion criteria were applied for the DM patients studied: 1) 18 to 25 years old; 2) a regular menstrual cycle of $\sim 27$ to 32 days; 3 ) a history of menstrual pain longer than 12 months; and 4) using the visual analog scale $^{21}$ (VAS, $0=$ not at all, $10=$ the worst imaginable pain), the self-assessed severity of the average menstrual pain over the previous six months was at least higher than 5. The inclusion criteria for the HC were similar to those for the DM, except that the controls had almost no pain during menses $(\mathrm{VAS}=0)$. Exclusion criteria included current pregnancy or breastfeeding, organic pelvic disease, alcohol or drug abuse, and formal diagnosis of psychiatric conditions. Urinary luteinizing hormone tests were performed to verify experimentally when participants were at their periovulatory phase (i.e., days 12-16 of the menstrual cycle). Ethics approval was granted by the Human Research Ethics Committee of South China Normal University. Signed informed consent was obtained from participants before the initial assessment was conducted. Participants were informed that they could withdraw from the study at any time. 
Sixty-one otherwise healthy participants with DM and 58 $\mathrm{HC}$ matched for age, education, calendar age, and gynecological age participated in this study. Five participants were excluded because they did not believe they were playing Cyberball with real participants (two from the DM group and three HC). Thus, all further analyses are based on data from 114 participants (59 DM and $55 \mathrm{HC}$ ). The participants' demographics are described in Table 1. Data are presented as mean $(\mathrm{M}) \pm \mathrm{SD}$. There were no between-group differences for any of the demographic variables.

\section{Social pain manipulation}

Cyberball, which is a well-established computerized balltossing game, was used to induce social pain. ${ }^{15,22}$ In this procedure, participants were tested individually but were led to believe that they were playing with two other university students from other laboratories connected through the internet. However, the other players did not exist, and the game was simulated by a computer program. Cyberball involves being rejected by strangers, which is likely less impactful than rejection by acquaintances. Therefore, as previously described, ${ }^{23-25}$ to increase the validity of Cyberball and to increase the impact of social exclusion, prior to beginning, groups of three participants (including one participant of the study and two "actors") engaged in a 10-minute group interaction session in which groups members' pictures were taken and photographs of two virtual players of each interaction session were shown to the participants throughout the game. DM and HC participants were randomly assigned to one of the two conditions (inclusion vs exclusion). Thus, the present study was a $2 \times 2$, between-subjects design. Each condition comprised 60 throws and took approximately four minutes. In the included condition, participants received the ball an equal number of times as other players throughout the game. In the exclusion condition, participants received only three out of the 60 throws before they were excluded, receiving no further throws.

\section{Physical pain sensitivity assessment Cold pressor task}

Cold pain threshold and tolerance were assessed by the well-known cold pressor task. ${ }^{26}$ The assessment device is a machine for cooling water through a compressor, which has a large insulated container divided by wire mesh into two compartments, with ice on one side and ice-free water on the other. The water, circulated by a submerged pump, was maintained at $1{ }^{\circ} \mathrm{C}$. The experimenter instructed the participant to put and keep her left hand in the compartment of ice-free water. As previously described, ${ }^{27}$ participants were asked 1) to tell the researcher when they first felt a sensation that they would call pain on any part of the hand that was submerged in the water (cold pain threshold, measured in seconds) and 2) to remove their hand from the water at any time but to leave their hand in the water for as long as they could. The time when participants withdrew their hand from the water indicated their cold pain tolerance, measured in seconds. ${ }^{16}$ The experimenter ended the assessment when the participant took her hand out of the water or after three minutes had elapsed. ${ }^{28}$

\section{Pressure algometer}

Pressure pain threshold and pain tolerance were measured using a pressure algometer (Wagner FPX 50, Wagner Instruments, Greenwich, CT, USA). This device assesses the amount of pressure applied to a muscle and has been used as one method of assessing pain sensitivity in the exclusion literature. ${ }^{12,19}$ It was applied perpendicularly to the skin and lowered at a rate of $\sim 5$ $\mathrm{kPa}$ per second until the pain threshold or tolerance limit was reached, as indicated by participants' verbal report. As previously described, ${ }^{12}$ pain threshold and pain tolerance measurements

Table I Participants' demographic characteristics

\begin{tabular}{lll}
\hline Variables & DM (n = 59) & HC (n = 55) \\
\hline Age, years & $20.03 \pm 1.75$ & $19.93 \pm 1.60$ \\
Age at menarche & $12.42 \pm 1.48$ & $12.78 \pm 1.18$ \\
Years of menstruating & $7.61 \pm 2.05$ & $7.15 \pm 1.88$ \\
Days of one menstrual cycle & $30.49 \pm 2.72$ & $29.91 \pm 2.47$ \\
Menstrual pain experience & & 0.157 \\
Age of the first menstrual pain & $14.57 \pm 1.97$ & - \\
Pain history, years & $5.32 \pm 2.61$ & - \\
VAS & $6.66 \pm 1.45$ & - \\
\hline
\end{tabular}

Note: Data are presented as mean \pm SD.

Abbreviations: DM, dysmenorrhea; HC, healthy female controls; VAS, visual analog scale. 
were taken at the first dorsal interosseous muscle (i.e., behind the first knuckle of the index finger) of the participant's right hand. For the pain threshold, participants were instructed to say "now" when they first felt pain due to the pressure increase. For pain tolerance, participants were instructed to say "stop" when the pain became too uncomfortable to continue. At this point, the algometer was immediately stopped and automatically recorded the amount of pressure applied prior to its cessation. ${ }^{19}$

\section{Psychological assessment}

\section{Positive- and negative-affect scales (PANAS)}

The Chinese version of the PANAS, ${ }^{29}$ with well-documented psychometric properties, ${ }^{30}$ was used to assess the mood of the participants before and after the social pain manipulation. This measure includes 20 items, 10 measuring positive affects and 10 measuring negative affects. Items are rated on a five-point scale ranging from 1 ("very slightly or not at all") to 5 ("extremely"). Higher scores in the positive affect subscale reflect a more positive mood, while higher scores in the negative-affect subscale reflect a more negative mood. In this study, Cronbach's alphas were 0.79 and 0.82 for positive and negative affects, respectively.

\section{Fear of negative evaluation scale (FNE)}

The FNE ${ }^{31}$ measures participants' concerns about being evaluated unfavorably by others before and after the social pain manipulation. The brief Chinese version of the FNE evaluating psychometric characteristics ${ }^{32}$ was used. This measure includes 12 items (e.g., "I worry about what other people will think of me even when I know it doesn't make any difference") that are rated on a five-point scale ranging from 1 ("not at all characteristic of me") to 5 ("extremely characteristic of me"). Higher scores reflect experiencing a higher degree of apprehension at the prospect of being evaluated negatively. In this study, the Cronbach's alpha for the overall scale was 0.87 .

\section{Self-Esteem Scale (SES)}

To assess overall feelings of self-worth and self-acceptance, participants completed the Rosenberg's SES. ${ }^{33}$ We used the validated Chinese version. ${ }^{34}$ This measure includes 10 items evaluated on a four-point scale ranging from 1 ("strongly agree") to 4 ("strongly disagree"). Higher scores reflect experiencing greater self-esteem. In this study, the Cronbach's alpha for the overall scale was 0.79 .

\section{Procedure}

After arriving at the lab, participants were seated in front of a computer screen and asked to complete a thorough consent form. All psychological questionnaires (including PANAS, FNE, and SES) were processed, after which the participants' baseline measures of pain threshold and tolerance were recorded. As described previously, ${ }^{19}$ the order of measurement of threshold and tolerance was counterbalanced. Participants had their threshold or tolerance measured first, took a 90-second break, and then continued with the other measure. Next, groups of three participants (including one participant of the study and two "actors") engaged in a 10-minute group interaction session, after which participants were assigned to three rooms individually and began the Cyberball game. Participants and experimenters were in a double-blind state, namely, participants were told that the purpose of the study was to examine the effect of imagination on mission performance; the experimenter did not know who among the participants the DM was. The pressure algometer test was applied immediately after each condition ended. Immediately after the end of the pressure stimulation, the cold pressor test was conducted. The order of measurement of pressure pain and cold pain was counterbalanced. Next, participants completed the PANAS, FNE, and SES questionnaires again (Figure 1). At the end of the experiment, participants were asked if they believed they were playing the Cyberball game with the other two participants. Finally, each participant received 30 (RMB) as compensation and were thoroughly debriefed about the purpose of the study.

\section{Statistical analysis}

The Full Information Maximum Likelihood method ${ }^{35}$ was used to handle missing data. Analyses were conducted using the SPSS for Windows Version 20 statistical package (IBM Corporation, Armonk, NY, USA). Analysis of variance (ANOVA) tests were used to assess differences in demographic characteristics between study groups and differences in pain sensitivity and psychological assessment between study conditions. As previously described, ${ }^{12,19}$ we ran analyses using baseline measures of pain threshold and tolerance as a covariate. Results of all analyses were considered significant at the $p<0.05$ level.

\section{Results \\ Pre-Cyberball pain sensitivity and psychological assessment}

The participants' baseline pain sensitivity and psychological assessments (Table 2) showed no between-group differences for any variables prior to the Cyberball experiment. 


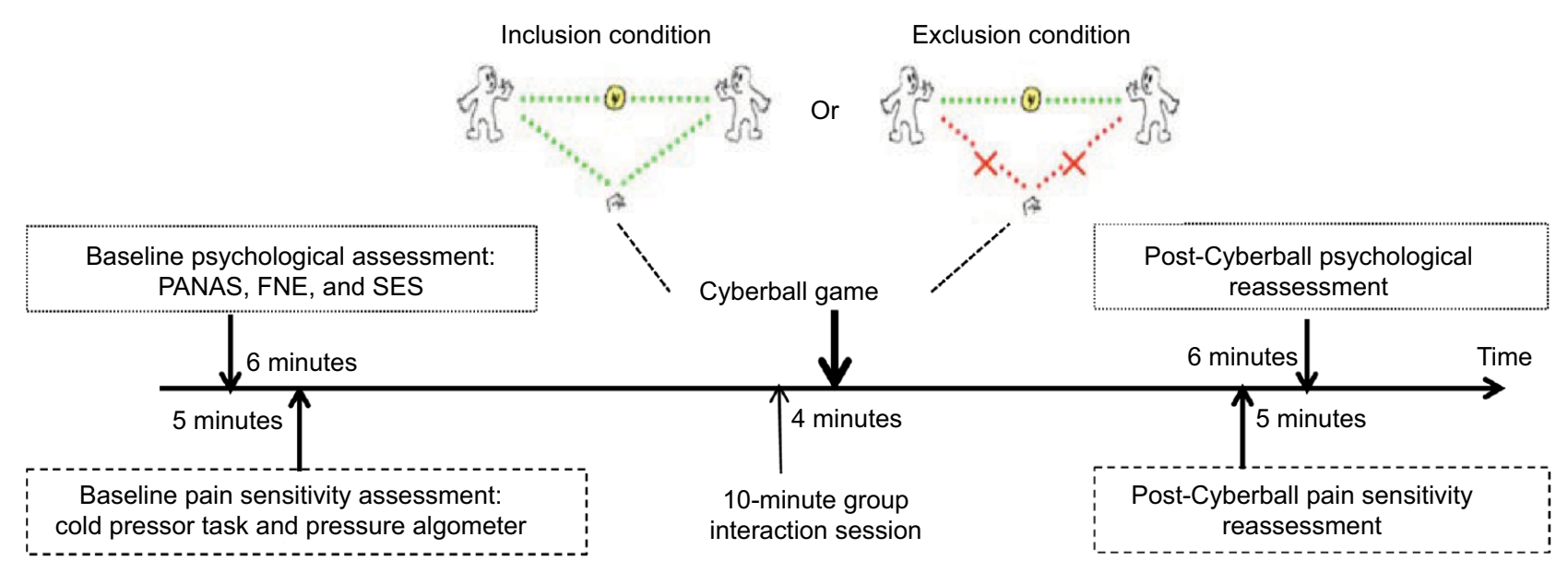

Figure I Experimental procedure schematic. All psychological questionnaires were processed, after which the participants' baseline measures of pain threshold and tolerance were recorded. Next, groups of three participants engaged in a 10-minute group interaction session, then DM and HC participants were randomly assigned to one of two Cyberball conditions (inclusion vs exclusion). Immediately after the end of the Cyberball game, the pain sensitivity retest was conducted. Next, participants completed the PANAS, FNE, and SES questionnaires again. The order of measurement of pain sensitivity (threshold and tolerance) and the order of measurement of pressure pain and cold pain were counterbalanced.

Abbreviations: DM, dysmenorrhea; FNE, fear of negative evaluation scale; HC, healthy female controls; PANAS, positive- and negative-affect scales; SES, Rosenberg selfesteem scale

Table 2 Baseline pain sensitivity and psychological characteristics

\begin{tabular}{|c|c|c|c|c|c|}
\hline \multirow[t]{2}{*}{ Variables } & \multicolumn{2}{|l|}{ DM } & \multicolumn{2}{|l|}{ HC } & \multirow[t]{2}{*}{$P$ (two-tailed) } \\
\hline & Inclusion $(n=30)$ & Exclusion $(n=29)$ & Inclusion $(n=26)$ & Exclusion $(n=29)$ & \\
\hline \multicolumn{6}{|l|}{ Cold pain } \\
\hline Threshold & $7.95 \pm 5.66$ & $10.41 \pm 9.23$ & $9.31 \pm 7.41$ & $7.59 \pm 5.87$ & 0.420 \\
\hline Tolerance & $|9.98 \pm 8.4|$ & $23.49 \pm 11.91$ & $28.90 \pm 30.33$ & $25.51 \pm 23.23$ & 0.408 \\
\hline \multicolumn{6}{|l|}{ Pressure pain } \\
\hline Threshold & $3.49 \pm 1.75$ & $3.44 \pm 1.68$ & $3.86 \pm 2.16$ & $3.30 \pm 1.48$ & 0.690 \\
\hline Tolerance & $7.60 \pm 3.07$ & $7.69 \pm 4.13$ & $8.41 \pm 4.09$ & $6.54 \pm 2.71$ & $0.27 I$ \\
\hline \multicolumn{6}{|l|}{ PANAS } \\
\hline Negative affect & $10.50 \pm 1.93$ & $10.86 \pm 1.79$ & $10.65 \pm 2.17$ & $10.41 \pm 1.62$ & 0.812 \\
\hline Positive affect & $17.00 \pm 5.30$ & $15.59 \pm 6.33$ & $16.73 \pm 7.32$ & $16.31 \pm 6.05$ & 0.838 \\
\hline FNE & $38.67 \pm 8.85$ & $43.48 \pm 6.69$ & $38.85 \pm 7.77$ & $41.07 \pm 6.81$ & 0.060 \\
\hline SES & $31.33 \pm 3.89$ & $30.03 \pm 4.02$ & $30.23 \pm 4.86$ & $30.86 \pm 5.22$ & 0.681 \\
\hline
\end{tabular}

Notes: Data are presented as mean $\pm \mathrm{SD} ; P$, the comparison result between the four listed experimental conditions.

Abbreviations: DM, dysmenorrhea; FNE, fear of negative evaluation scale; HC, healthy female controls; PANAS, positive- and negative-affect scales; SES, Rosenberg SelfEsteem Scale.

\section{Post-Cyberball psychological assessment}

ANOVA was conducted separately for the positive and negative affects experienced by participants subjected to either social inclusion or social exclusion for each of the two groups, DM or HC. The statistical analysis revealed that the effect of social experience was significant $[F(1,110)=29.25$, $p=0.000]$, with, as expected, the exclusion condition resulting in a higher negative affect than inclusion in both DM and $\mathrm{HC}$ groups (Figure 2A). The inclusion condition resulting in a higher positive affect than exclusion in both $\mathrm{DM}$ and $\mathrm{HC}$ groups $[F(1,110)=28.04, p=0.000]$.
A similar analysis of FNE data again showed the predicted interaction [ANOVA; $F(1,110)=4.16, p<0.05$ ]. For DM participants, simple effect analyses revealed that Cyberball exclusion resulted in greater concern at being evaluated unfavorably by others, compared to inclusion (exclusion: $\mathrm{M}=44.55, \mathrm{SD}=5.79$; inclusion: $\mathrm{M}=33.60, \mathrm{SD}=6.51$; $F(1,111)=35.37, p=0.000)$. Furthermore, in contrast to inclusion conditions, which did not show a difference between DM and HC groups, there was a significant difference between DM and HC participants under exclusion conditions (inclusion: $F(1,111)=0.04, p>0.05$; exclusion: 
A

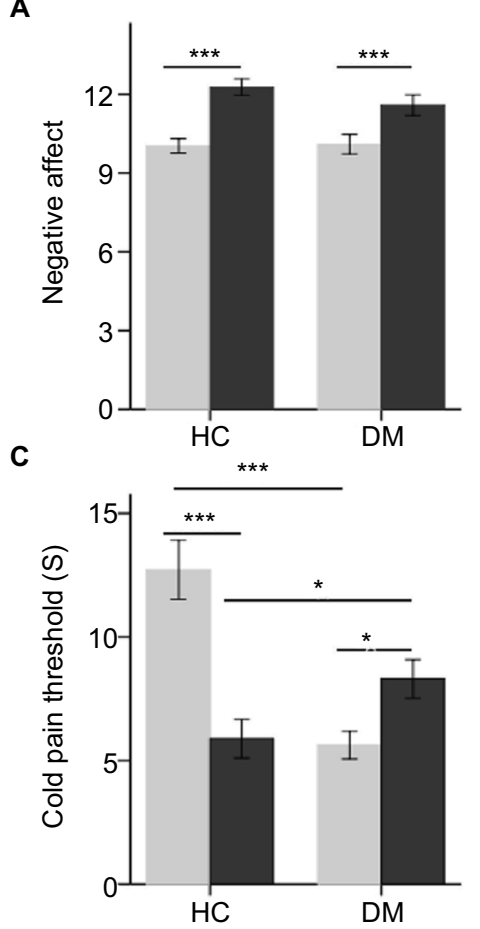

B

D
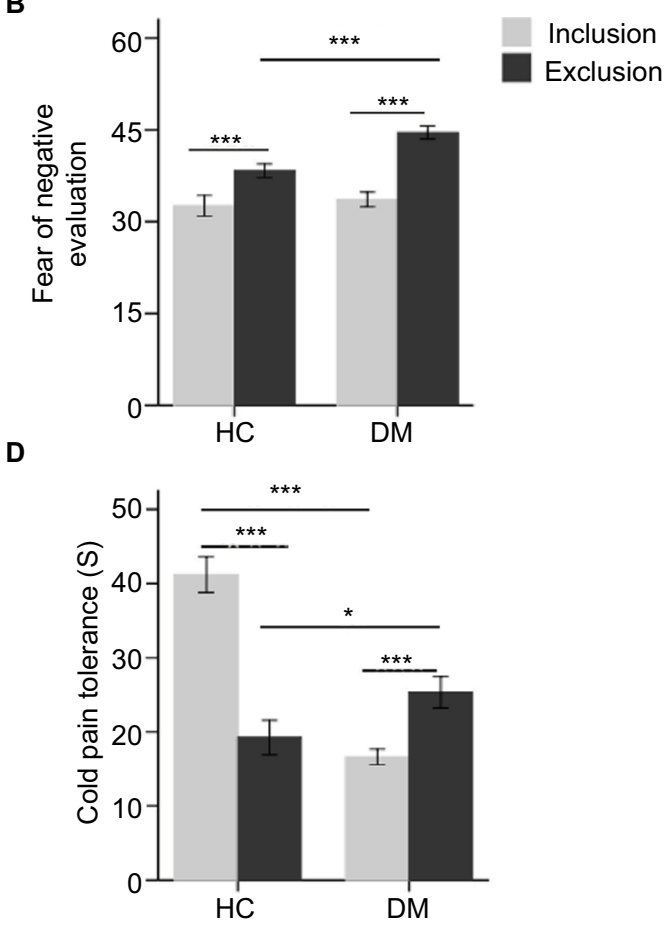

Figure 2 Post-Cyberball cold pain sensitivity and psychological perspectives in the DM and HC groups. (A) Post-Cyberball negative-affect score as a function of social experience; (B) post-Cyberball fear of negative evaluation score as a function of social experience; (C) post-Cyberball cold pain threshold score as a function of social experience; (D) post-Cyberball cold pain threshold and tolerance score as a function of social experience. ${ }^{* * *} p<0.00 \mathrm{I},{ }^{*} p<0.05$.

Abbreviations: DM, dysmenorrhea; $\mathrm{HC}$, healthy female controls;

$F(1,111)=8.84, p<0.005)$. Thus, DM participants subjected to social exclusion were more concerned at being evaluated unfavorably by others compared to $\mathrm{HC}$ (DM: $\mathrm{M}=44.55$, SD $=5.79$; $\mathrm{HC}: \mathrm{M}=38.31, \mathrm{SD}=6.51$; Figure 2B).

ANOVA showed that there were no significant differences in self-esteem scores between the inclusion and exclusion conditions in the DM and $\mathrm{HC}$ groups.

\section{Post-Cyberball pain sensitivity Cold pain}

The post-Cyberball cold pain threshold data were assessed by analysis of covariance (ANCOVA), using pre-Cyberball cold pain threshold data as a covariate, which revealed the predicted interaction $[F(1,109)=32.78, p<0.0005]$. Simple effect analyses showed that, in DM participants, Cyberball exclusion resulted in a higher cold pain threshold than inclusion (exclusion: $\mathrm{M}=8.30, \mathrm{SD}=4.22$; inclusion: $\mathrm{M}=5.62, \mathrm{SD}=3.07 ; F(1,111)=5.16, p<0.05)$. However, conversely, exclusion resulted in a lower cold pain threshold than inclusion in $\mathrm{HC}$ participants (exclusion: $\mathrm{M}=5.88$, SD $=4.19$; inclusion: $\mathrm{M}=12.72, \mathrm{SD}=6.09 ; F(1,111)=29.22$, $p=0.0005)$. Furthermore, the cold pain threshold was higher in DM than $\mathrm{HC}$ participants under exclusion conditions (DM: $\mathrm{M}=8.30, \mathrm{SD}=4.22 ; \mathrm{HC}: \mathrm{M}=5.88, \mathrm{SD}=4.19 ; F(1$, $111)=4.09, p<0.05)$. However, the cold pain threshold of DM participants was lower than $\mathrm{HC}$ under social inclusion conditions (DM: $\mathrm{M}=5.62, \mathrm{SD}=3.07 ; \mathrm{HC}: \mathrm{M}=12.72, \mathrm{SD}$ $=6.09 ; F(1,111)=32.31, p=0.0005 ;$ Figure $2 \mathrm{C})$.

We next conducted ANCOVA on post-Cyberball cold pain tolerance data with the pre-Cyberball cold pain tolerance as a covariate, which revealed the predicted interaction $(F(1$, $109)=83.60, p<0.0005)$. Simple effect analyses revealed that Cyberball exclusion resulted in higher cold pain tolerance than inclusion in DM participants (exclusion: $\mathrm{M}=25.32$, $\mathrm{SD}=11.47$; inclusion: $\mathrm{M}=16.62, \mathrm{SD}=5.74 ; F(1,111)=$ $8.43, p=0.005)$. However, in contrast, exclusion resulted in lower cold pain tolerance than inclusion in $\mathrm{HC}$ (exclusion: $\mathrm{M}=19.22, \mathrm{SD}=12.50$; inclusion: $\mathrm{M}=41.19, \mathrm{SD}=12.29$; $F(1,111)=46.09, p=0.0005$; Figure 2D).

Moreover, not only did DM individuals differ from $\mathrm{HC}$, but both showed changes compared to the baseline situation. Using one-sample $t$-tests, we compared the pain sensitivity between baseline (Time 1) and post-Cyberball (Time 2). Cyberball inclusion significantly decreased cold 
pain threshold and tolerance at Time 2 compared to Time 1 in DM participants (threshold: $t(29)=-2.99, p=0.006$; tolerance: $t(29)=-3.10, p=0.004)$. In contrast, cold pain threshold and tolerance remained unchanged between Time 1 and Time 2 after social exclusion in DM participants (threshold: $t(28)=-1.35, p=0.189$; tolerance: $t(28)=1.42, p=$ $0.166)$. Interestingly, exclusion caused hypersensitivity and thus reduced the cold pain threshold and tolerance at Time 2 compared to Time 1 in HC participants (threshold: $t$ (28) $=-2.581, p=0.01$; tolerance: $t(28)=-2.13, p=0.042)$. However, inclusion increased cold pain threshold and tolerance in the HC group (threshold: $t(25)=2.72, p=0.012$; tolerance: $t(25)=2.44, p=0.022)$.

\section{Pressure pain}

There were no significant differences in post-Cyberball pain threshold and tolerance between inclusion and exclusion conditions in either the DM or the HC participants. The post-Cyberball pressure pain threshold and tolerance data were assessed by ANCOVA, using pre-Cyberball pressure pain threshold or tolerance data as a covariate, which did not reveal the predicted interaction (threshold: $F(1,109)=0.228$, $p=0.634$; tolerance: $F(1,109)=2.874, p=0.093)$. The main effect of conditions (exclusion vs inclusion) did not differ significantly (threshold: $F(1,109)=0.870, p=0.353$; tolerance: $F(1,109)=0.414, p=0.522)$. The main effect of groups (DM vs HC) did not differ significantly as well (threshold: $F(1,109)$ $=0.441, p=0.508$; tolerance: $F(1,109)=0.083, p=0.773)$.

\section{Discussion}

Studies on HC participants show that pain-triggered emotion modulates the pain process, ${ }^{36}$ but it is still unclear whether a painful experience actually modulates the sensation of pain. The current study shows that, in contrast to $\mathrm{HC}$, socially excluded individuals with DM demonstrate an increase in both pain threshold and tolerance to pain in response to cold stimulation. However, similar to fibromyalgia patients, ${ }^{16}$ having experienced social inclusion, DM sufferers demonstrate decreased pain threshold and tolerance. These results suggest that the modifying effect of social experience on the pain process differs in DM compared to HC participants, which unambiguously supports our hypothesis that an individual's prior pain experience differentially modulates the effects of social pain on the sensitivity to and tolerance of physical pain. Therefore, individual pain experience appears to be an important moderator that can lead to either intensification or abatement of physical pain after social exclusion, confirming an overlap between the physical and social pain processes.
Cyberball exclusion results in decreased cold pain threshold and tolerance compared to inclusion in $\mathrm{HC}$, supporting the notion that the positive emotions associated with inclusion reduce pain perception, while correspondingly the negative emotions linked with exclusion induce pain hypersensitivity. ${ }^{37-39}$ This result provides us with new behavioral evidence that improves our understanding of the relationships between social pain and a specific type of physical pain.

The current study also suggests that, having experienced social exclusion, DM sufferers are more fearful of being evaluated unfavorably by others, and accordingly are more eager to be positively evaluated. One possible explanation is that the unique combination of both acute and chronic pain associated with DM causes long-term stress, which in turn leads to emotional sensitivity and anxiety. ${ }^{40}$

We observed no significant differences in self-esteem scores between the inclusion and exclusion conditions for either the DM or the HC group, similar to other studies showing that social exclusion does not affect the level of self-esteem of the participants. ${ }^{41,42}$ It is likely that, since the majority of people have defensive mechanisms in place, isolated threats such as occasional social exclusion can be deflected without damaging self-esteem. ${ }^{43}$

Notably, unlike the result with cold pain, neither DM nor HC participants showed a significant difference in post-Cyberball pressure pain threshold and tolerance between the inclusion and exclusion conditions. This discrepancy might be explained by differences in afferent pathways involved in cold-induced and pressure-induced pain. The detection of skin surface temperatures is achieved by axons terminating at lamina I of the spinal dorsal horn, and the highest correlation with subjective ratings of temperature is located in the orbitofrontal and anterior insular cortex. ${ }^{44}$ In contrast, pressure exerted on the skin activates nociceptive afferents in several tissues (e.g., intraepidermal nerve endings); enhanced pain sensitivity to blunt pressure is likely due to alterations in descending pathways from the brainstem. ${ }^{45}$ Alternatively, the discrepancy might be due to differences in the pain assessment procedures. Thus, the cold pain assessment tested the participant's whole hand, while the pressure pain assessment involved only a single finger; such differences in the location and tissue depth of the pain sensation, as well as the nature of the experimental pain stimulus itself, could result in inconsistency in pain sensitivity. ${ }^{46,47}$

\section{Conclusion}

The present study suggests that pain modulation in DM sufferers shows a disrupted response to social experience. Though the relatively small sample size may result in low power and a consequent inability to detect some significant 
effects, our study indicates that previous pain experience affects the regulation of physical pain sensation and certain associated psychometric properties by social pain. It is worth noting that we are unable to comment on causality due to the correlation nature of this study. Although a formal diagnosis of psychiatric condition was an exclusion criterion, we cannot rule out the possibility that the DM group differs in mental health parameters. Additional measures evaluating mental health of participants are necessary. The effect of mental health (such as depression, anxiety, and somatization) on pain sensitivity requires further research.

\section{Acknowledgments}

The present study was supported by grants from the National Natural Science Foundation of China (31771219, 31171355), the Natural Science Foundation of Guangdong Province (2014A030313418, 2014A030313440), and the Science and Technology Division of Guangzhou (201607010320).

\section{Disclosure}

The authors report no conflicts of interest in this work.

\section{References}

1. Jamieson DJ, Steege JF. The prevalence of dysmenorrhea, dyspareunia, pelvic pain, and irritable bowel syndrome in primary care practices. Obstet Gynecol. 1996;87(1):55-58.

2. Iacovides S, Avidon I, Baker FC. What we know about primary dysmenorrhea today: a critical review. Hum Reprod Update. 2015;21(6):762-778.

3. Wei SY, Chao HT, Tu CH, et al. Changes in functional connectivity of pain modulatory systems in women with primary dysmenorrhea. Pain. 2016;157(1):92-102.

4. Kutch JJ, Tu FF. Altered brain connectivity in dysmenorrhea: pain modulation and the motor cortex. Pain. 2016;157(1):5-6.

5. Dun WH, Yang J, Yang L, et al. Abnormal structure and functional connectivity of the anterior insula at pain-free periovulation is associated with perceived pain during menstruation. Brain Imaging Behav. 2017;11(6):1787-1795.

6. Bajaj P, Bajaj P, Madsen H, Arendt-Nielsen L. A comparison of modality-specific somatosensory changes during menstruation in dysmenorrheic and nondysmenorrheic women. Clin J Pain. 2002;18(3):180-190.

7. Tu FF, Epstein AE, Pozolo KE, Sexton DL, Melnyk AI, Hellman KM. A noninvasive bladder sensory test supports a role for dysmenorrhea increasing bladder noxious mechanosensitivity. Clin J Pain. 2013;29(10):883-890.

8. Granot M, Yarnitsky D, Itskovitz-Eldor J, Granovsky Y, Peer E, Zimmer EZ. Pain perception in women with dysmenorrhea. Obstet Gynecol. 2001;98(3):407-411.

9. Pitangui ACR, Gomes MRD, Lima AS, Schwingel PA, Albuquerque APD, de Araujo RC. Menstruation disturbances: prevalence, characteristics, and effects on the activities of daily living among adolescent girls from Brazil. J Pediatr Adol Gynec. 2013;26(3):148-152.

10. Eryilmaz G, Ozdemir F, Pasinlioglu T. Dysmenorrhea prevalence among adolescents in eastern Turkey: its effects on school performance and relationships with family and friends. $J$ Pediatr Adol Gynec. 2010;23(5):267-272.
11. Eisenberger NI. Social pain and the brain: controversies, questions, and where to go from here. Annu Rev Psychol. 2015;66:601-629.

12. DeWall CN, Baumeister RF. Alone but feeling no pain: effects of social exclusion on physical pain tolerance and pain threshold, affective forecasting, and interpersonal empathy. J Pers Soc Psychol. 2006;91(1):1-15.

13. Eisenberger NI. The pain of social disconnection: examining the shared neural underpinnings of physical and social pain. Nat Rev Neurosci. 2012;13(6):421-434.

14. Williams KD, Jarvis B. Cyberball: A program for use in research on interpersonal ostracism and acceptance. Behav Res Methods. 2006;38(1):174-180.

15. Eisenberger NI, Lieberman MD, Williams KD. Does rejection hurt? An FMRI study of social exclusion. Science. 2003;302(5643):290-292.

16. Canaipa R, Castro-Caldas A, Moreira JM, Pimentel-Santos F, Branco JC, Treister R. Impaired pain modulation in fibromyalgia patients in response to social distress manipulation. Clin J Pain. 2017;33(7):611-619.

17. Macdonald G, Floyd K, Kingsbury R. Does physical pain augment anxious attachment? J Soc Pers Relat. 2006;23(2):291-304.

18. Eisenberger NI, Jarcho JM, Lieberman MD, Naliboff BD. An experimental study of shared sensitivity to physical pain and social rejection. Pain. 2006;126(1-3):132-138.

19. Bernstein MJ, Claypool HM. Social exclusion and pain sensitivity: why exclusion sometimes hurts and sometimes numbs. Pers Soc Psychol Bull. 2012;38(2):185-196.

20. Borsook TK, MacDonald G. Mildly negative social encounters reduce physical pain sensitivity. Pain. 2010;151(2):372-377.

21. Tu CH, Niddam DM, Yeh TC, et al. Menstrual pain is associated with rapid structural alterations in the brain. Pain. 2013;154(9):1718-1724.

22. Williams KD, Cheung CKT, Choi W. Cyberostracism: effects of being ignored over the Internet. J Pers Soc Psychol. 2000;79(5):748-762.

23. McQuaid RJ, McInnis OA, Matheson K, Anisman H. Distress of ostracism: oxytocin receptor gene polymorphism confers sensitivity to social exclusion. Soc Cogn Affect Neurosci. 2015;10(8):1153-1159.

24. Rotge J-Y, Lemogne C, Hinfray S, et al. A meta-analysis of the anterior cingulate contribution to social pain. Soc Cogn Affect Neurosci. 2015;10(1):19-27.

25. Eisenberger NI. Meta-analytic evidence for the role of the anterior cingulate cortex in social pain. Soc CognAffect Neurosci. 2015;10(1):1-2.

26. von Baeyer CL, Piira T, Chambers CT, Trapanotto M, Zeltzer LK. Guidelines for the cold pressor task as an experimental pain stimulus for use with children. J Pain. 2005;6(4):218-227.

27. Andrews NE, Meredith PJ, Strong J. Adult attachment and reports of pain in experimentally-induced pain. Eur J Pain. 2011;15(5):523-530.

28. Frías MT, Shaver PR. The moderating role of attachment insecurities in the association between social and physical pain. $J$ Res Pers. 2014;53:193-200.

29. Huang L, Yang T, Li Z. Applicability of the positive and negative affect scale in Chinese applicability of the positive and negative affect scale in Chinese. Chin Mental Health J. 2003;17(1):54-56.

30. Spencer-Rodgers J, Peng KP, Wang L. Dialecticism and the co-occurrence of positive and negative emotions across cultures. $J$ Cross Cult Psychol. 2010;41(1):109-115.

31. Collins KA, Westra HA, Dozois DJ, Stewart SH. The validity of the brief version of the fear of negative evaluation scale. J Anxiety Disord. 2005;19(3):345-359.

32. Yen JY, Yen CF, Chen CS, Wang PW, Chang YH, Ko CH. Social anxiety in online and real-life interaction and their associated factors. Cyberpsychol Behav Soc Netw. 2012;15(1):7-12.

33. Rosenberg M. Society and the Adolescent Self-Image. Princeton, NJ: Princeton University Press; 1965.

34. Ye SQ, Yu L, Li KK. A cross-lagged model of self-esteem and life satisfaction: gender differences among Chinese university students. Pers Indiv Differ. 2012;52(4):546-551. 
35. Graham JW. Missing data analysis: making it work in the real world. Annu Rev Psychol. 2009;60:549-576.

36. Rainville P, Bao QVH, Chretien P. Pain-related emotions modulate experimental pain perception and autonomic responses. Pain. 2005;118(3):306-318.

37. Rhudy JL, Williams AE, Mccabe KM, Thu MA, Nguyen V, Rambo P. Affective modulation of nociception at spinal and supraspinal levels. Psychophysiology. 2005;42(5):579-587.

38. Wunsch A, Philippot P, Plaghki L. Affective associative learning modifies the sensory perception of nociceptive stimuli without participant's awareness. Pain. 2003;102(1-2):27-38.

39. Canaipa R, Treister R, Lang M, Moreira JM, Castro-Caldas A. Feeling hurt: pain sensitivity is correlated with and modulated by social distress. Clin J Pain. 2016;32(1):14-19.

40. Jorum E. Analgesia or hyperalgesia following stress correlates with emotional behavior in rats. Pain. 1988;32(3):341-348.

41. Twenge JM, Catanese KR, Baumeister RF. Social exclusion and the deconstructed state: time perception, meaninglessness, lethargy, lack of emotion, and self-awareness. J Pers Soc Psychol. 2003;85(3):409-423.
42. Blackhart GC, Nelson BC, Knowles ML, Baumeister RF. Rejection elicits emotional reactions but neither causes immediate distress nor lowers self-esteem: a meta-analytic review of 192 studies on social exclusion. Pers Soc Psychol Rev. 2009;13(4):269-309.

43. Blackhart GC, Knowles ML, Nelson BC, Baumeister RF. Rejection elicits emotional reactions but neither causes immediate distress nor lowers self-esteem: a meta-analytic review of 192 studies on social exclusion. Pers Soc Psychol Rev. 2009;13(4):269-309.

44. Nomoto S, Shibata M, Iriki M, Riedel W. Role of afferent pathways of heat and cold in body temperature regulation. Int J Biometeorol. 2004;49(2):67-85.

45. Treede RD, Rolke R, Andrews K, Magerl W. Pain elicited by blunt pressure: neurobiological basis and clinical relevance. Pain 2002;98(3):235-240.

46. Sherman JJ, LeResche L. Does experimental pain response vary across the menstrual cycle? A methodological review. Am J Physiol ReguI Integr Comp Physiol. 2006;291(2):R245-R256.

47. Vincent K, Tracey I. Sex hormones and pain: the evidence from functional imaging. Curr Pain Headache Rep. 2010;14(5):396-403.

\section{Journal of Pain Research}

\section{Publish your work in this journal}

The Journal of Pain Research is an international, peer reviewed, open access, online journal that welcomes laboratory and clinical findings in the fields of pain research and the prevention and management of pain. Original research, reviews, symposium reports, hypothesis formation and commentaries are all considered for publication.

\section{Dovepress}

The manuscript management system is completely online and includes a very quick and fair peer-review system, which is all easy to use. Visit http://www.dovepress.com/testimonials.php to read real quotes from published authors. 